methods like permeability or liquid displacement can be successfully applied to both inorganic and polymeric membranes. The main difference with static methods is that only active pores are described with these two last methods.

Once the porous structure of the membrane has been properly characterised, appropriate phenomenological equations can be applied to describe transport phenomena. Generally the driving force used for liquid filtration is obtained by a pressure gradient $\Delta p$ applied between feed and permeate faces of the membrane. According to linear non-equilibrium thermodynamics, it has been traditional to characterise the permeability of a porous membrane with respect to transmembrane convective and diffusive transport of a neutral solute solution in terms of four parameters: hydraulic permeability $L_{p}$, diffusive permeability $\omega$ and two reflective coefficients $\sigma_{\mathrm{d}}$ and $\sigma_{\mathrm{f}}$ respectively for solvent and solute. The hydraulic permeability is a measure of the ability to transport a volume of liquid under the action of the pressure difference across the membrane. This property is expressed as the proportionality coefficient between the volume flux $J_{v}$ and the transmembrane pressure $\Delta p$, when the solute is absent:

$$
L_{p}=J_{v} / \Delta p \text { for pure solvent }
$$

This definition also applies in the presence of a solute when the membrane is freely permeable to the solute or when the rejected entities in the feeding solution do not induce an osmotic phenomenon (micro- and ultrafiltration). In other cases, in particular for microporous membranes (nanofiltration), the flux is affected by osmotic pressure differences across the membrane. The volumetric flux expression for a solution generalises from the previous equation:

$$
J_{\mathrm{v}}=L_{\mathrm{p}}\left(\Delta p-\sigma_{\mathrm{d}} \Delta \Pi\right)
$$

in which $\Delta \Pi$ is the osmotic pressure difference at the membrane/solution interface due to the solute and $\sigma_{\mathrm{d}}$ is the Staverman osmotic reflexion coefficient.

The diffusive permeability $\omega$ characterises the transmissibility of the membrane for the solute when the volumetric flux is constrained to be zero:

$$
\omega=J_{\mathrm{s}} / \Delta \Pi \text { for } J_{\mathrm{v}}=0
$$

in which $J_{s}$ is the transmembrane molar flux for the solute. In general, solute transport by both convection and diffusion occurs and the two contributions are assumed to be additive, giving the general equation for transmembrane solute flux:

$$
J_{\mathrm{s}}=\left(1-\sigma_{\mathrm{f}}\right) J_{\mathrm{v}} C+\omega R T \mathrm{~d} c / \mathrm{d} x
$$

in which $R T \mathrm{~d} c / \mathrm{d} x=\Delta \Pi$ (van't Hoff law), $C$ is the average solute concentration on both sides of the membrane and $\sigma_{\mathrm{f}}$ is the Staverman filtration coefficient for the solute.

This approach of mass transport can be adapted to idealised pore structures in order to predict or interpret observed filtration performance. In practice however, most of the membranes do not correspond to idealised pore structures. Moreover, mass transport can be affected by the occurrence of secondary phenomena such as interaction of solutes or solvent with the membrane material and concentration polarisation. It follows that the volumetric flux can be written in a different manner:

$$
J_{\mathrm{v}}=\Delta p / \eta R_{\mathrm{tot}}
$$

in which $R_{\mathrm{tot}}$ is the sum of the resistances to the transport across the membrane and $\eta$ the viscosity of the liquid. The various resistances contribute to different extents to the total resistance. In the ideal case only the intrinsic membrane resistance is involved. During an actual separation process additional resistances are responsible for flux decline. They can be attributed to phenomena such as accumulation of retained solutes on the feed side (concentration polarisation and gel layer formation) or to the penetration and immobilisation of solutes inside the pores (adsorption and pore blocking). It follows that the modelling of transport across a porous layer becomes very complex when applied to a specific problem involving a real fluid.

\section{Transport of Permanent and Condensable Gases in Zeolite Membranes}

Klaas Keizer, Zeger A. E. P. Vroon, Henk Verweij \& Anthonie J. Burggraaf

University of Twente, Laboratory of Inorganic Materials Science, Faculty of Chemical Technology, PO Box 217, 7500AE Enschede, The Netherlands

High temperature zeolite membranes represent a new class of membranes with molecular sieve properties for distinct molecules. The main zeolite used for these types of membranes is MFI (silicalite, ZSM) with $5 \cdot 5 \AA$ pores but a few papers/patents have been concerned with zeolite A. In this contribution we concentrate on MFIzeolite.

A polycrystalline zeolite layer is grown on a porous $\alpha$-alumina support with $160 \mathrm{~nm}$ pores with a multi in-situ crystallisation process at temperatures between 100 and $180^{\circ} \mathrm{C}$. The two-step crystallisation process results in membranes with the best properties. The zeolite layer thickness varies between 2 and $7 \mu \mathrm{m}$ with crystallise between varying 0.1 and $1 \mu \mathrm{m}$, depending on 
synthesis temperature. MFI is three-dimensional zeolite so the polycrystalline material is suitable for transport, but it is possible that on grain boundaries some extra transport resistance is present.

Transport through these membranes is complex and depends not only on the size of molecules but also on their specific interaction with the zeolite. Consequently the temperature and pressure of the transport and/or separation process is important. There are three main types of gas molecules which have different behaviour for transport through these membranes:

1. Small gas molecules $(<4 \AA$ ) without interaction with the zeolite material. Examples are $\mathrm{H}_{2}, \mathrm{He}, \mathrm{CH}_{4}, \mathrm{~N}_{2}$ and $\mathrm{O}_{2}$. Here transport accords with Knudsen diffusion (square root mass ratios) and gases transport independently.

2. Small/medium sized molecules $(<5 \AA)$, which are condensable and show strong interaction with the zeolite material especially at low temperatures and high pressures. The transport of of these gas molecules is strongly interdependent. Examples are $\mathrm{CO}_{2}$ and all hydrocarbons from $\mathrm{C}_{3}$ and above.

3. Large molecules ( $>5 \AA$ ), which show size exclusion effects and do not adsorb strongly because of their size. This includes single- and di-branched aliphatic hydrocarbons. For the aromatic hydrocarbons benzene will transport faster than $o$ - and $m$-xylene but about the same as $p$-xylene and toluene because the kinetic diameter is similar.

To illustrate this complex transport some permselectivities and separation factors are shown in Table 1. Combinations of groups 1 and 2 (methane/ $n$-butane), and 2 and 3 are shown. In single gas experiments methane transports somewhat faster than $n$-butane $\left(P_{\alpha, \mathrm{me} / \mathrm{nb}}\right)$ especially at low temperatures. In mixtures $\left(\alpha_{\text {me/nb }}\right) n$-butane is much faster, because of high occupation of the zeolite pores with butane molecules. The transport is now adsorption controlled. At high temperatures ratios of single gases come close to separation factors.

In the combination $2 / 3$ single gas transport ratios and separation factors are comparable (see $P_{\alpha, n b / i b}$ and $\left.\alpha_{n b / i b}\right)$. The separation becomes much larger with a com-

\section{TABLE 1}

Permselectivites $(P)$ and Separation Factors $(\alpha)$ of $50 / 50$ Methane (me)/n-Butane (nb), nb/Isobutane (ib), Hexane (hex)/ 2,2-Dimethylbutane (dmb) and Para/Ortho-Xylene (p/o-xyl). The Flux of Pure Methane is 10 and $7 \mathrm{mmol} \mathrm{m}^{-2} \mathrm{~s}^{-1}$ at a Feed Pressure of $100 \mathrm{kPa}$ at 25 and $200^{\circ} \mathrm{C}$ Respectively

\begin{tabular}{rlllllr}
\hline$T$ & $P_{\alpha, \mathrm{me} / \mathrm{nb}}$ & $\alpha_{\mathrm{me} / \mathrm{nb}}$ & $P_{\alpha, \mathrm{nb} / \mathrm{ib}}$ & $\alpha_{\mathrm{nb} / \mathrm{ib}}$ & $\alpha_{\mathrm{hex} / \mathrm{dmb}}$ & $\alpha_{p / 0-\mathrm{xy} 1}$ \\
$\left({ }^{\circ} \mathrm{C}\right)$ & & & & & & \\
\hline 25 & 2.7 & 0.06 & 90 & 52 & 400 & 1 \\
200 & 1.2 & 0.7 & 11 & 11 & $>2000$ & $>20$ \\
\hline
\end{tabular}

bination of linear and di-branched molecules. The isomers hexane and 2,2-dimethylbutane show a separation factor of over 2000 at $200^{\circ} \mathrm{C}$ for example. With branched-aromatic hydrocarbons the transport is even more complex. Because of the size of the molecules, transport is lower than $1 \mathrm{mmol} \mathrm{m}^{-2} \mathrm{~s}^{-1}$ for $100 \mathrm{kPa}$ feed and zero permeate pressure. Separation occurs for the isomers of xylene $(>20)$.

\section{Fluid Transport in Porous Materials Characterised by NMR}

\section{Ken J. Packer}

Department of Chemistry, University of Nottingham, Nottingham NG7 2RD, UK

The NMR properties of spins in fluids contained in porous solids can reflect the porosity of the containing solid, its internal surface-to-volume ratio and aspects of the structure of the pore space. ${ }^{1}$ The NMR signal intensity measures porosity directly, as long as a calibration measurement is also made and the signal is determined under conditions which ensure that the effects of inhomogeneous internal magnetic fields and diffusion of molecules through these are removed. This is usually achieved by means of multiple-pulse echo sequences. The surface-to-volume ratio of the containing pore space affects the fluid spins through acting as a source of relaxation, be this spin-lattice (SLR) or transverse relaxation. Diffusion of the spin-bearing molecules to the interface is then an important relaxation mechanism. In the fast diffusion limit $[\rho a / D \ll 1]$ the SLR rate is given by

$$
R_{1}=\rho(S / V) \approx \rho / a
$$

where $a$ is a measure of the linear scale of the pore space, $\rho$ is the surface relaxation strength and $D$ the selfdiffusion coefficient. For slow diffusion the relaxation is multiexponential with the longest and dominant component having a relaxation rate given by

$$
R_{1}^{\text {long }} \approx\left(D / a^{2}\right)
$$

NMR can also characterise diffusion directly through use of the pulsed magnetic field-gradient spin echo (PGSE) experiment. ${ }^{2}$ The response of this experiment, in the limit of short gradient pulses, can be expressed as

$$
S(q, \Delta)=\iint \mathrm{d} x_{0} \mathrm{~d} x P\left(x_{0}\right) P\left(x, \Delta ; x_{0}\right) \exp \left\{i q\left(x-x_{0}\right)\right\}
$$

where $q$ is the gradient pulse area $\left(=\gamma \delta g_{x} / 2 \pi\right), \Delta$ the time over which transport is measured, $g_{x}$ the gradient (chosen to be along $x)$ and with $P\left(x_{0}\right)$ and $P\left(x, \Delta ; x_{0}\right)$, respectively, the probability density of starting positions $x_{0}$ and the conditional probability that spins will be 